\title{
El marketing a través de la segmentación, focalización y posicionamiento del mercado
}

\author{
Marketing through segmentation, targeting and market positioning \\ Ana Yuridia Morales Flores ${ }^{a}$, Omar Cruz Gómez ${ }^{b}$
}

\begin{abstract}
:
Marketing is the human process that identifies the needs of society for remuneration. On the other hand, segmentation is the division by groups or areas of people in which their needs are identified and the ways of satisfying them, thus elaborating complete and quality products. Taking the strategies of persuasion hand in hand with marketing to achieve a resounding success in our companies.
\end{abstract}

Keywords:

Marketing, segmentation, targeting, market positioning, persuasion

Resumen:

El mercadeo es el proceso humano que identifica las necesidades de la sociedad a cambio de la remuneración. Por otro lado, la segmentación es la división por grupos o áreas de personas de las cuales se identifican sus necesidades y las formas de satisfacerlas, elaborando productos completos y de buena calidad. Llevando así las estrategias de persuasión de la mano con el mercadeo para lograr un éxito rotundo en nuestras compañías.

Palabras Clave:

Marketing, segmentación, focalización, posicionamiento, persuasión.

\section{Marketing a través de la segmentación, focalización y posicionamiento del mercado}

La importancia de utilizar estrategias para la venta de productos y dar un buen servicio al cliente, son fundamentales para garantizar el marketing como exitoso.

Es necesario conocer sobre este tema porque a partir de aquí se puede generar la verdadera construcción de una organización o red de personas, que compartan la calidad de los productos, y así crear una seguridad financiera [1].

El éxito de empresas como Apple, se ha mantenido en gran parte su debido posicionamiento de nuevos productos en el mercado, mismo que, cada día espera más y mejor tecnología [2].

Construir una organización implica manejar ambientes competitivos, así como también a tener una actitud correcta y creer en nosotros mismos para poder visualizar el éxito en nuestra organización o empresa, es de vital importancia confiar en la industria, creer en el plan de compensación, compañía y productos y reflejar esto en nuestro mercadeo, lo anterior hará que el cliente desee adquirir nuestros productos y así crecerá nuestro negocio hasta llegar a ser de primer nivel [3].

Uno de los elementos decisivos del éxito de una empresa se debe a la capacidad de segmentar adecuadamente su mercado objeto. La segmentación es también, un esfuerzo por mejorar la precisión del marketing de una compañía, de la cual su esencia es: conocer realmente a los consumidores.

Para tener un buen funcionamiento de una empresa se necesita unidad, seguido de un plan de desarrollo en el cual se plasmen las ideas para que esta se fusione de acuerdo a los estándares previstos, objetivos y metas de lo que realmente se quiere lograr.

Autor de Correspondencia, Universidad Autónoma del Estado de Hidalgo, Escuela Superior de Huejutla, Email: ana_morales9717@uaeh.edu.mx 
Toda empresa debe estar complementada entre sí y hacer mercadotecnia, de modo que con diferentes estrategias se logre un alcance significativo en la economía, y al consumidor.

En el siguiente diagrama se explica de manera gráfica y sencilla el funcionamiento del Marketing a través de la segmentación, focalización y posicionamiento del mercado.

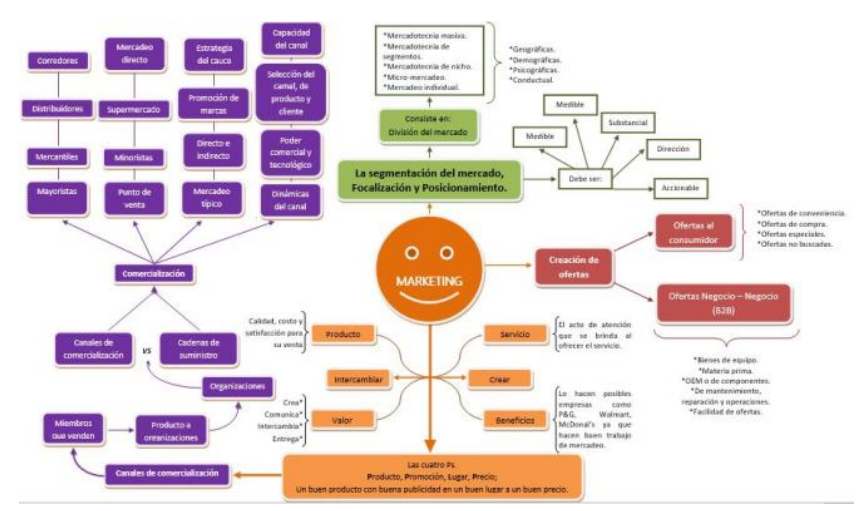

Figura 1. Diagrama de Marketing a través de la segmentación, focalización y posicionamiento del mercado.

Finalmente, ¿Qué es lo que hace o convence a las personas de que todo puede funcionar de la mejor manera? ¿De dónde nace el Marketing? La respuesta es simplemente una: Persuasión. Este término, que muchas veces es subestimado por quienes operan en las áreas de diseño y operación, es el núcleo y hará que todo lo demás funcione si es aplicado de forma correcta.

La persuasión no opera en la cabeza, sino en el estómago. En su artículo "6 técnicas de persuasión que absolutamente siempre funcionan", nos enfoca al marketing desde la perspectiva de la persuasión [4].

Concluyendo así que si lo que necesitas es llevar tu organización a otro nivel, la capacidad de argumentar y persuadir va de la mano generando así resultados de mercadeo satisfactorios.

\section{Referencias}

[1] M. Carpenter, J. F. Tanner and K. Collins, Management, Monterrey: ITESM, S.F.

[2] D. H., "Apple chief tim Cook Opts out 75 million dividend," 05 mayo 2012. [Online]. Available: www.guardian.co.uk.
[3] G. Jones and J. George, Admimnisttracipon contemporánea. Octava edición., México, DF.: Mc Graw Hill, 2014.

[4] F. García Pimentel, "6 técnicas de persuasión que absolutamente siempre funcionan (o por qué Trump se sale con la suya)," 03 Mayo 2019. [Online]. Available: https://www.entrepreneur.com/article/330034. 